\title{
ОЦЕНКА ПАРАМЕТРОВ КАЛЬЦИЙ-ФОСФОРНОГО ОБМЕНА У ПАЦИЕНТОВ С ДЕФИЦИТОМ ВИТАМИНА D
}

\author{
Катамадзе Н.Н., Шутова А.С., Фаргиева Х.Р., Поваляева А.А., Пигарова Е.А., Дзеранова Л.К., Глазьева В.С., \\ Жуков А.Ю., Беловалова И.М., Дедов И.И. \\ ФГБУ «НМИЦ эндокринологии» Минздрава России, Москва \\ Московский государственный университет имени М.В. Ломоносова, России, Москва
}

Для поддержания оптимального уровня содержания кальция и фосфора в крови требуется нормальное количество витамина $\mathrm{D}$, который имеет важное значение для адекватной минерализации костей, сокращения мышц, нервной проводимости и функционирования клеток организма человека. Дефицит витамина D приводит к снижению эффективности абсорбции кальция и фосфора в кишечнике. Поддержание адекватного уровня кальция и фосфора в крови обеспечивает оптимальную секрецию паратгормона (ПТГ) паращитовидными железами, который, в свою очередь, стимулирует образование активного метаболита - 1,25 дигидроксивитамина $\mathrm{D}_{3}$ в почках.

ЦЕЛЬ: оценка тактики врачей по определению параметров кальций-фосфорного обмена у пациентов с дефицитом витамина D.

МЕТОДЫ: мы провели национальный опрос врачей через Российскую ассоциацию эндокринологов, в результатае которого были получены данные о том, как часто врачи оценивают состояние кальцийфосфорного обмена путем измерения общего содержания кальция, ионизированного кальция, кальция с поправкой на альбумин, фосфор и ПтГ у пациентов с дефицитом витамина D. B опросе приняли участие 704 врача, специализирующихся в различных областях. 80\% из них составляли эндокринологи, 7,6\% и 3,9\% - акушеры-гинекологи и терапевты, а 8,5\% - врачи других специальностей.

PЕЗУЛЬТАТЫ: согласно результатам опроса, доля врачей, оценивающих содержание общего кальция, ионизированного кальция, кальция, скорректированного на уровни альбумина, фосфора и ПтГ у пациентов с дефицитом витамина D, составила 70,5, 69,1, 41,9, 65,6 и 77,2\% соответственно. Опрос показал, что чаще всего и более полно среди опрошенных врачей оценивают параметры кальций-фосфорного обмена эндокринологи (92,3\%).

ВЫВОдЫ: оценка параметров кальций-фосфорного обмена до определения уровня витамина D перед его назначением с целью коррекции необходима для исключения различных форм гиперпаратиреоза и предупреждения возможного развития гиперкальциемии. Наше исследование показало, что эндокринологи чаще оценивают уровни кальция и фосфатов в сыворотке крови, чем врачи других специальностей. 